ISSN: 0514-7336

DOI: https://doi.org/10.14201/zephyrus201779221231

\title{
SOBRE LA PREGNANCIA EN LA UTILIZACIÓN DEL RELIEVE EN LAS CUEVAS DE BÉDEILHAC (ARIĖGE, FRANCIA) Y DE EL PINDAL (ASTURIAS, ESPAÑA)
}

\section{About the prominence in the use of natural accidents in the caves of Bédeilhac (Ariège, France) and El Pindal (Asturies, Spain)}

\author{
Georges Sauvet* y María GonZÁlez-PumariegA** \\ * Centre de Recherche et d'Études de l'Art Préhistorique (CREAP-Cartailhac). Maison des Sciences de l'Homme \\ et de la Société de Toulouse (USR 3414). Université de Toulouse Jean-Jaurés. Allées Antonio Machado. \\ Toulouse (France). Correo-e: georges.sauvet@sfr.fr \\ ** Consejería de Educación y Cultura. Principado de Asturias. Cueva El Pindal. \\ Correo-e: maria.glez-pumariegasolis@asturias.org
}

Recepción: 16/11/2016; Revisión: 3/02/2017; Aceptación: 23/03/2017

Resumen: El aprovechamiento de relieves naturales es una característica bien conocida del arte parietal paleolítico, aunque no se le concede generalmente la atención que merece. En esta nota, queremos añadir al repertorio dos ejemplos inéditos que provienen de las cuevas de Bédeilhac (Ariège, Francia) y de El Pindal (Asturias, Espańa). En ambos casos, se trata de relieves ligeramente separados de la pared que fueron acondicionados para representar cabezas de animales indeterminados. La intervención humana es mínima pues consiste en la adición de un ojo grabado y algunos trazos de pigmento rojo. La similitud de los tratamientos en estas dos cuevas atestigua motivaciones compartidas y una comunidad de pensamiento. Así, los relieves utilizados de Bédeilhac y El Pindal se suman a las numerosas convergencias ya conocidas entre ambos sitios, particularmente la presencia de claviformes pirenaicos en El Pindal, y confirman una proximidad cultural muy fuerte entre los Pirineos centrales y el occidente de la región cantábrica durante el Magdaleniense.

Palabras-clave: Magdaleniense; Pirineos franceses; Región Cantábrica; intercambios culturales; Psicología de la Forma.

AвsтRAct: The utilisation of natural reliefs is well known in the rock art of the Upper Palaeolithic, but it is often underestimated. In this note, two unpublished examples coming from the caves of Bédeilhac (Ariège, France) and El Pindal (Asturias, Spain) are described. In both cases, they are reliefs slightly separated from the wall, which have the shape of a natural animal head. The only human intervention consisted in the addition of an engraved eye and some traces of red pigment. The similarity of treatment in the two caves suggests a commonality of thought. Thus the reliefs used in Bédeilhac and El Pindal must be added to the numerous convergences already known between the two sites, among which the presence of Pyrenean claviforms in El Pindal, and confirms a strong cultural proximity between the French Pyrenees and the western side of the Cantabrian region during the Magdalenian period.

Key words: Magdalenian; French Pyrenees; Cantabrian region; cultural exchanges; Gestalt psychology. 


\section{Introducción}

El arte parietal paleolítico plantea preguntas apasionantes sobre su significado y su función en el seno de las sociedades de cazadores-recolectores, si bien dichas cuestiones quedan fuera de alcance de la metodología arqueológica. A pesar de ello, estas representaciones, pintadas o grabadas, figurativas o abstractas, escondidas o visibles, repartidas por centenares de cuevas y abrigos a través de Europa, constituyen una herramienta formidable a disposición de los arqueólogos que deseen abordar la problemática de las relaciones sociales y culturales entre los grupos y su evolución en el espacio y en el tiempo a lo largo del Paleolítico superior.

Combinando diferentes enfoques, técnicos, temáticos, compositivos y estilísticos, y utilizando diferentes métodos de análisis, es posible constatar la existencia de relaciones entre grupos separados a veces por más de mil kilómetros. Estos vínculos, evidenciados por las similitudes tanto del arte parietal como del arte mueble, confirman los datos arqueológicos relativos a la difusión de aquellas materias primas más apreciadas, entre las cuales figuran, obviamente, los indispensables materiales silíceos, así como las conchas utilizadas para la fabricación de ornamentos (Álvarez Fernández, 2005).

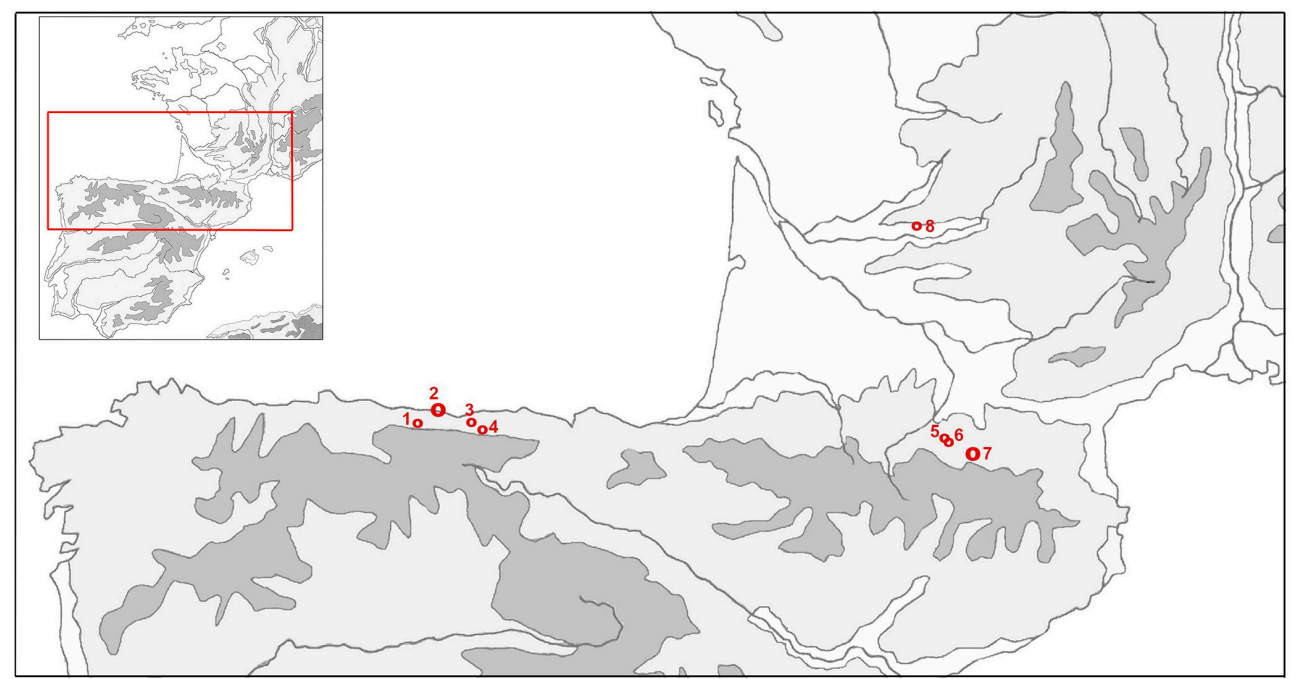

FIG. 1. Mapa del so de Francia y de la cornisa cantábrica con indicación de los sitios mencionados en el texto: 1. El Bosque; 2. El Pindal; 3. Altamira; 4. El Castillo; 5. Le Tuc d'Audoubert; 6. Les Trois-Frères; 7. Bédeilhac; 8. Bernifal.
En muchos casos, los intercambios materiales y culturales entre grupos humanos muy alejados parecen mucho más intensos de lo que se podría estimar para distancias que se pueden recorrer en algunas horas de marcha. Esos intercambios a larga distancia suponen la existencia de relaciones duraderas entre grupos alejados, en el seno de las llamadas "redes de intercambio".

En trabajos anteriores, reunimos numerosos argumentos para demostrar la existencia de estas redes, que incluyen el suroeste de Francia y la cornisa cantábrica (Sauvet et al., 2008, 2014). Así, hemos defendido particularmente la existencia de vínculos preferentes entre yacimientos de la región del Ariège, en los Pirineos centrales, y Asturias, en el occidente de la Región Cantábrica (Sauvet, 2014). En este trabajo, no volveremos sobre aquellos argumentos ya expuestos, pero queremos aportar uno suplementario para ilustrar la estrecha relación que los magdalenienses mantenían con las cuevas. Hablaremos de lo que generalmente se designa con la expresión "aprovechamiento de relieves naturales", aunque realmente constituya un elemento fundamental de su interés por el medio subterráneo.

Los dos ejemplos inéditos que vamos a describir en esta nota proceden de las cuevas de Bédeilhac (Ariège, Francia) y de El Pindal (Asturias, España), cavidades que encierran representaciones paradigmáticas del arte magdaleniense (Fig. 1). El tema de este trabajo no sorprenderá a aquellos convencidos del carácter "pirenaico" de una gran parte del arte de El Pindal. 


\section{Cabeza de animal en relieve natural de la cueva de Bédeilhac}

La cueva de Bédeilhac tiene un yacimiento magdaleniense en su inmenso vestíbulo y también varias áreas de ocupación situadas cerca de los principales locus de arte parietal (Robert, 1946). En la Sala Terminal, a $750 \mathrm{~m}$ de la entrada, los magdalenienses se instalaron sobre una suerte de terraza de techo bajo, fácil de iluminar y calentar. Alrededor de esta zona, las paredes y los techos están repletos de grabados y pinturas, con algunas figuras bien acabadas que responden a los cánones magdalenienses más clásicos, aunque, sobre todo, con grafismos inacabados, muchas veces indescifrables. Además, numerosos restos rojos, más o menos difusos, particularmente
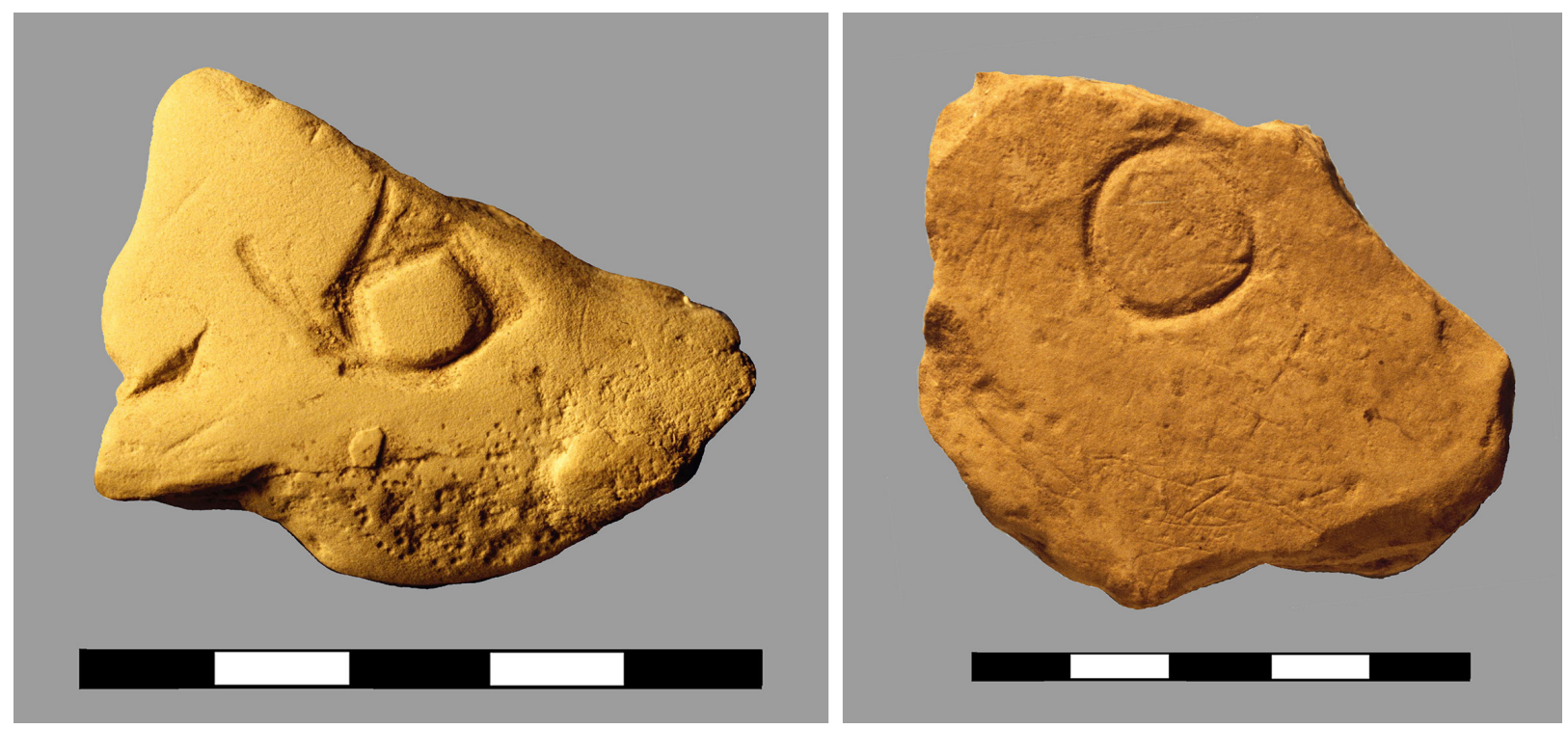

FIG. 2. Bédeilhac: ejemplos de "cabezas en ángulo"; obsérvese la importancia concedida al ojo, a menudo el único elemento que certifica la intención figurativa del autor.
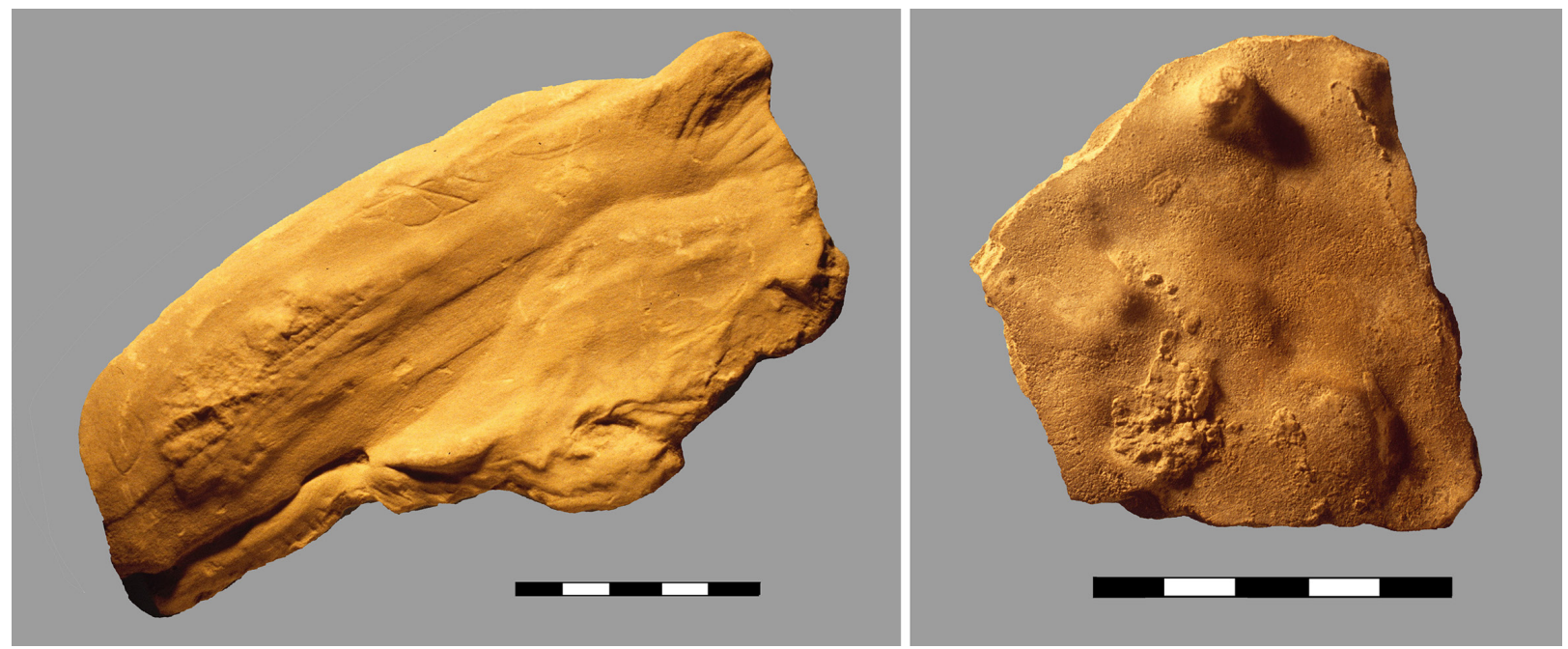

FIG. 3. Bédeilhac: plaquetas de arenisca acondicionadas como cabezas de caballo y bisonte; ejemplos de plaquetas que figuran animales identificables. Nótese la utilización perfectamente controlada de una pequeña prominencia estalagmitica para figurar el cuerno del bisonte. 
en las zonas bajas, pueden ser interpretados como rastros dejados por el frotamiento de los cuerpos o de las ropas. Las excavaciones proporcionaron herramientas líticas y óseas del Magdaleniense medio pirenaico, así como numerosos restos faunísticos que demuestran la estancia relativamente duradera de un pequeño grupo y no simples idas y venidas desde el yacimiento de entrada. En este sector se han descubierto varios centenares de plaquetas grabadas, correspondiéndose un gran número de ellas con lo que el comandante Octobon describió como "cabezas en ángulo" (Octobon, 1938). Se trata de pequeńas plaquetas de arenisca blanda, con un contorno natural utilizado para evocar un hocico animal. La intervención humana es generalmente mínima: un ojo, un ollar, algunos trazos para figurar la barba bastan para confirmar la intención del autor (Sauvet, 2004). De nuevo, encontramos en estas plaquetas figuras de un arte muy acabado frente a muchas caricaturas mediocres (Fig. 2). El enorme abanico de habilidades técnicas revela una pluralidad de autores con desigual talento para el grabado, lo que sugiere la idea de que cada miembro del grupo pudiera grabar su propia plaqueta por motivos personales. Así, consideramos estos grabados como ofrendas o exvotos y este sector de la Sala Terminal como un lugar consagrado a una práctica ritual (Sauvet, 2004).

Uno de los aspectos más sorprendentes de estas obras parietales y muebles es el fuerte vínculo que mantienen con el soporte mineral. Algunas de las piezas mobiliares más bellas muestran una aguda capacidad de observación de las particularidades morfológicas del soporte. Así, un pequeño abultamiento estalagmítico fue aprovechado para representar el cuerno de un bisonte (Fig. 3). En el arte parietal, un bisonte situado en el techo rasante de la Sala Terminal demuestra que tal perspicacia

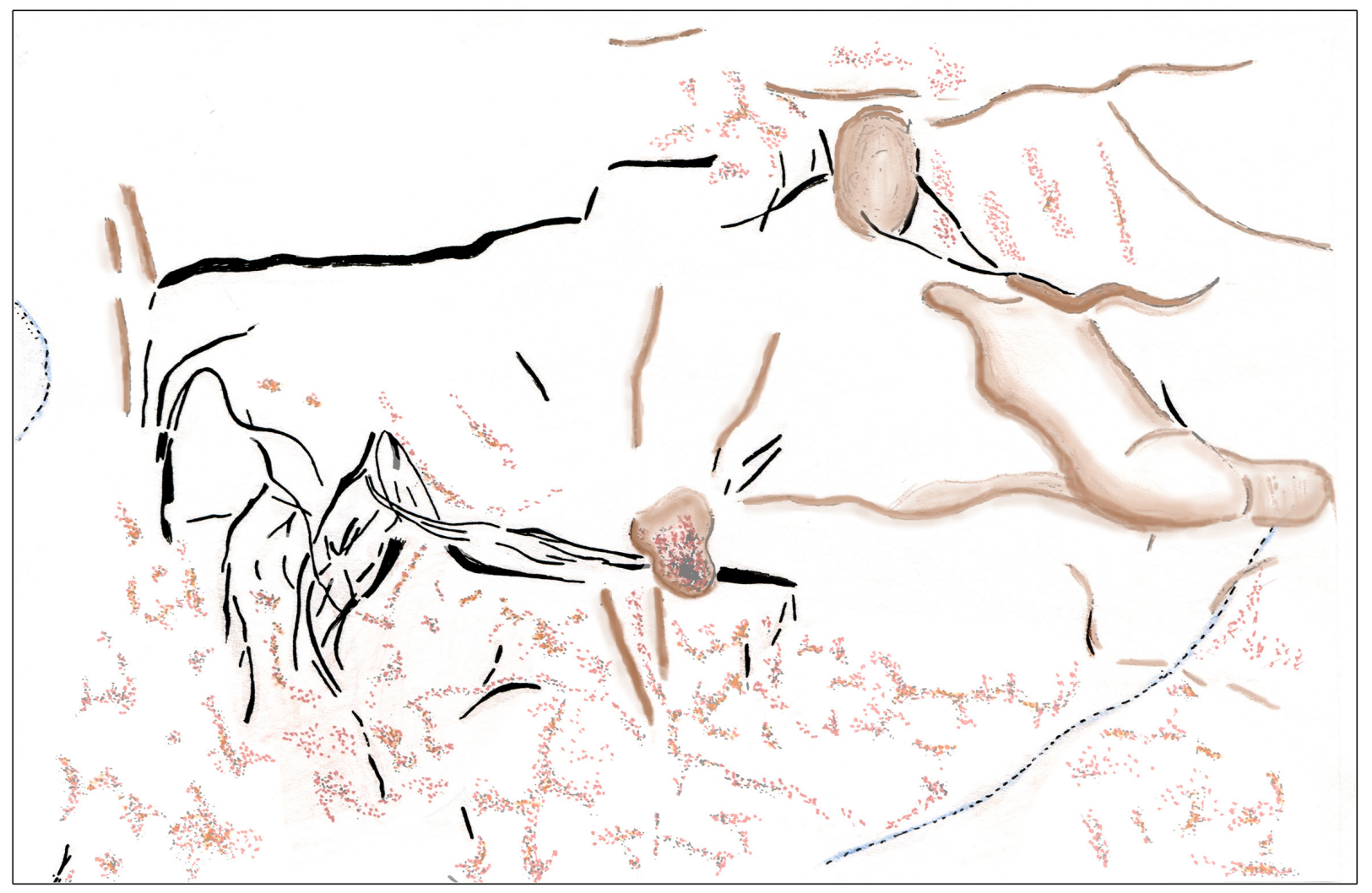

FIG. 4. Bédeilhac, Sala Terminal: bisonte, en parte grabado y en parte natural, situado en un techo bajo; la cabeza es un relieve y el cuerno, un cordón de calcita, igual que la pata anterior. 
no es fruto del azar sino objeto de una búsqueda sistemática (Fig. 4).

El relieve natural objeto de esta nota está situado en el borde del techo bajo. Inédito hasta ahora, este saliente constituye lo que en el arte de las catedrales llamaríamos una gárgola. Su apariencia de cabeza animal no escapó al magdaleniense, quien lo tiñó de ocre rojo y le grabó profundamente un ojo fusiforme, descartando así toda ambigüedad sobre su intención de animar el resalte rocoso (Fig. 5).

Los restos rojos se reparten por toda la cara aunque son particularmente abundantes en las zonas prominentes, como la frente y el hocico, y se parecen más a toques difusos que a trazos con una función definida. El grabado del ojo es particularmente vigoroso: las curvas que definen la órbita se desdoblan y son más claras que el relieve oscuro interior, que figura el globo ocular, lo que favorece la animación de esta cabeza. La forma natural del resalte rocoso ha deparado aquí el contorno de una gruesa cabeza de animal indeterminado, cuya identificación es arriesgada. En efecto, no parece que el autor haya tenido la voluntad de precisar una especie concreta.

\section{Cabeza de animal en relieve natural de la cueva de El Pindal}

La cueva de El Pindal es un conducto lineal de cerca de $300 \mathrm{~m}$, prolongado por una galería estrecha y caótica de unos $290 \mathrm{~m}$, prácticamente desprovista de vestigios arqueológicos. La concentración principal de arte parietal está localizada en la pared derecha y se extiende entre los metros 185 y 270. $\mathrm{El}$ inventario de las representaciones figurativas ha aumentado de 21 (Alcalde del Río et al., 1911) a 30 (González-Pumariega, 2011), entre los cuales 14 son bisontes y 8 caballos, aunque las figuras más célebres son un mamut rojo y un pez grabado. Sin perjuicio de la cronología del conjunto, hay consenso en la edad magdaleniense de la totalidad de los grabados.

Igual que en Bédeilhac y otras muchas cuevas, se encuentran marcas rojas dispersas a lo largo de las paredes y sobre los espeleotemas de gran parte de la cavidad, lo que muestra que los hombres prehistóricos invistieron la totalidad del espacio disponible.

En la cueva, hay varias utilizaciones de relieves, pero la mayoría de ellas son discretas y afectan sólo a ciertos segmentos de las figuras. Una de las más interesantes es una cabeza de bisonte construida a partir de una arista rocosa redondeada, que es una de las últimas figuras descubiertas (González-Pumariega, 2005). La arista define la línea frontal, el hocico y el principio de la barba de una cabeza en perfil izquierdo. Un ligero relieve interior dibuja el contorno del maxilar (Fig. 6). El relieve está situado entre dos concavidades, en la parte inferior de un conjunto de pinturas rojas constituido por varios grupos de puntuaciones, una serie de claviformes sobre un colgante rocoso, un bisonte rojo y grabado y una cabeza de caballo roja (Fig. 7). Sobre él sólo se grabaron el ojo, ovalado, con trazos múltiples, dos cuernos en perspectiva y algunos trazos lineales para figurar la barba (Fig. 6b). Los trazos grabados arañaron la pátina superficial, por lo que se muestran en gris oscuro, color de la roca basal. Este bisonte, visto de tres cuartos, parece saliendo de la pared. A pesar de la discreción de los trazos grabados, aparece como una pieza esencial de la composición, centrada en los claviformes.

En este contexto, el reconocimiento de un segundo relieve natural adquiere todo su valor. Este se encuentra en el extremo derecho del panel principal (Fig. 8). Varios trazos rojos habían sido ya catalogados en esta zona (González-Pumariega, 2011), entre los cuales se pueden mencionar líneas que parecen pertenecer a una figura animal mal conservada $-n .^{\circ}$ 7- y una silueta de mamut - n. ${ }^{\circ} 10-$, figura que ya existía en el calco de Breuil (Alcalde del Río et al., 1911), aunque su correcta identificación es reciente (Balbín et al., 1999).

El relieve ahora identificado, como en el caso de Bédeilhac, es un resalte natural que evoca una cabeza de animal en perfil derecho, menos destacada de la pared que en la cueva pirenaica. La parte que se separa del soporte dibuja una frente abombada, una línea fronto-nasal rectilínea, un hocico redondeado y un maxilar bastante voluminoso (Fig. 9). 


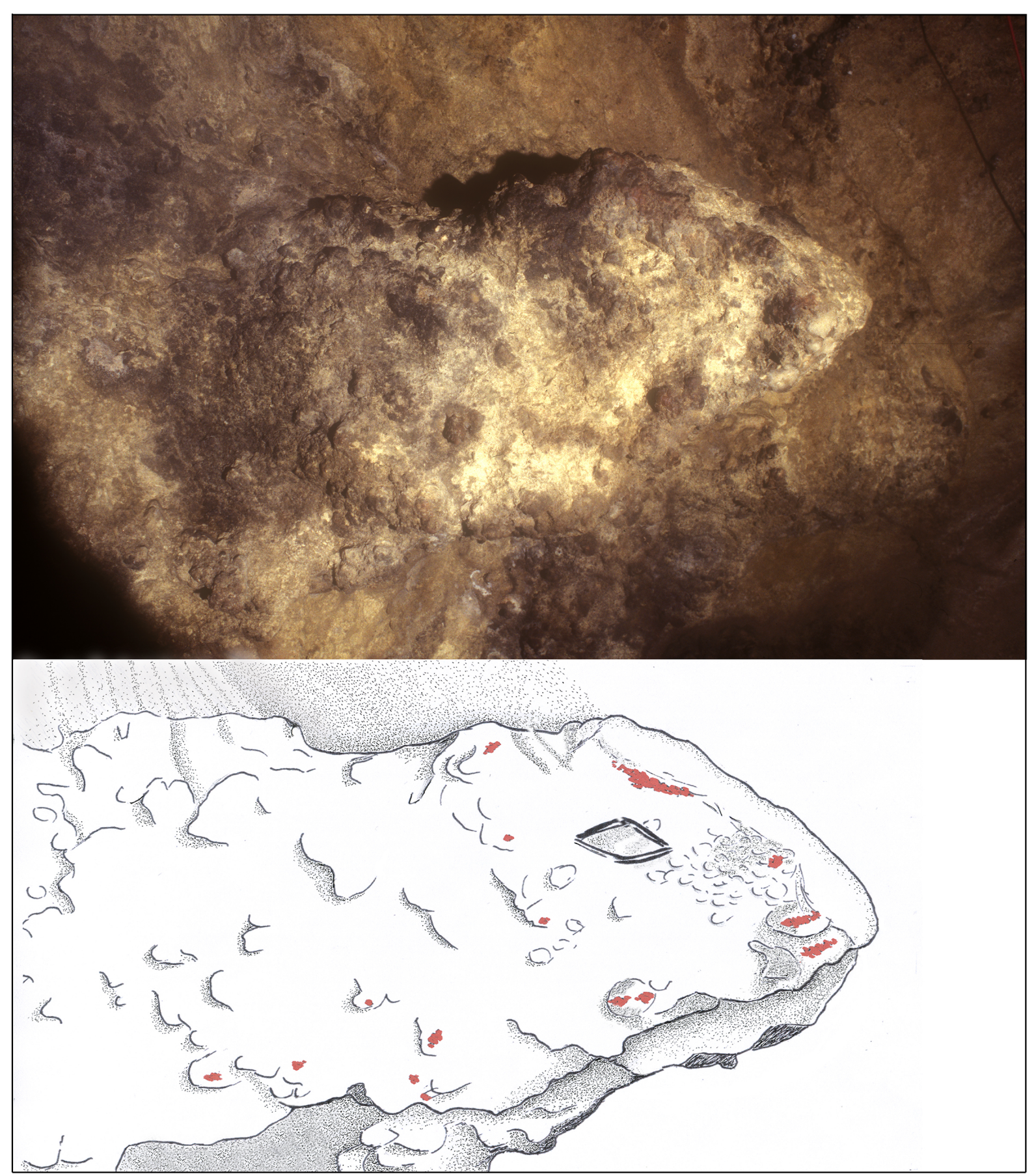

FIG. 5. Bédeilhac, Sala Terminal: fotografía y calco de un resalte rocoso acondicionado como cabeza animal mediante la adición de un ojo grabado y algunos trazos rojos.

Algún magdaleniense aprovechó la oportunidad y procedió de la misma manera que su homólogo de Bédeilhac. Le bastó con añadir un ojo ovalado bastante pequeño, en un grabado poco profundo, pero indudable, y un trazo rojo paralelo a la testuz. La similitud de este relieve con una cabeza de animal 

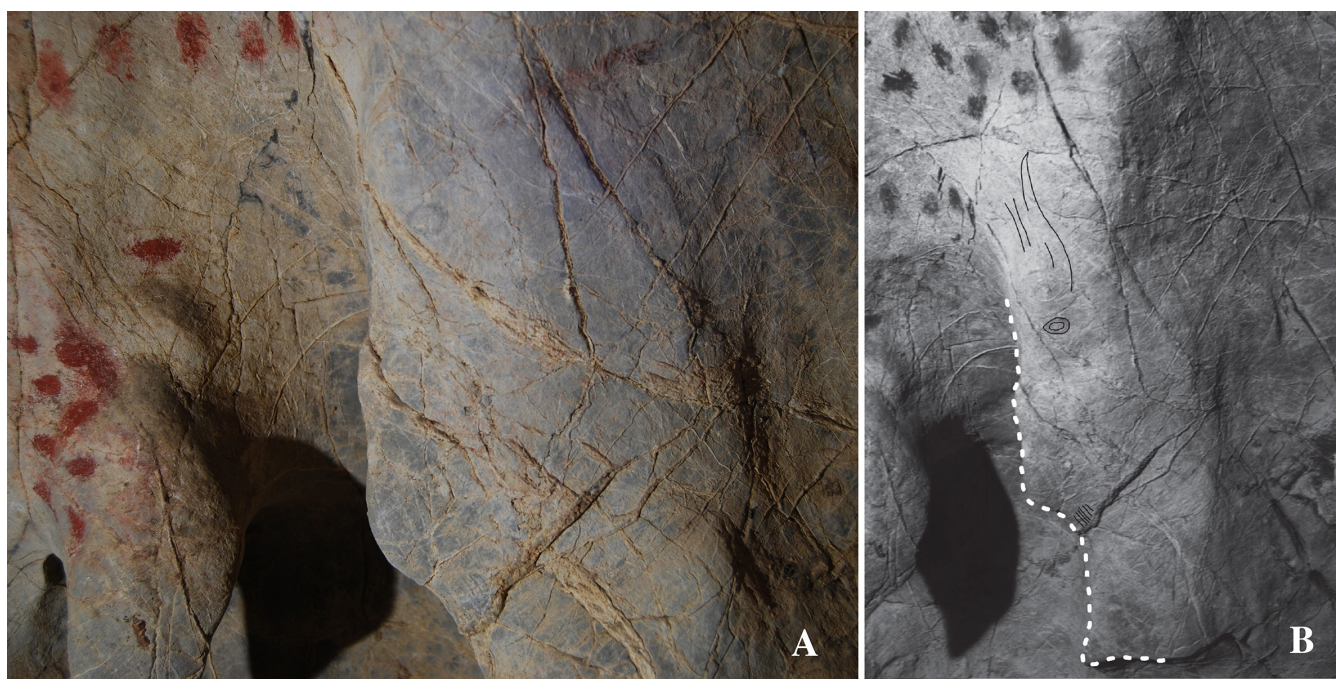

Fig. 6. El Pindal: cabeza de bisonte sobre un resalte rocoso.

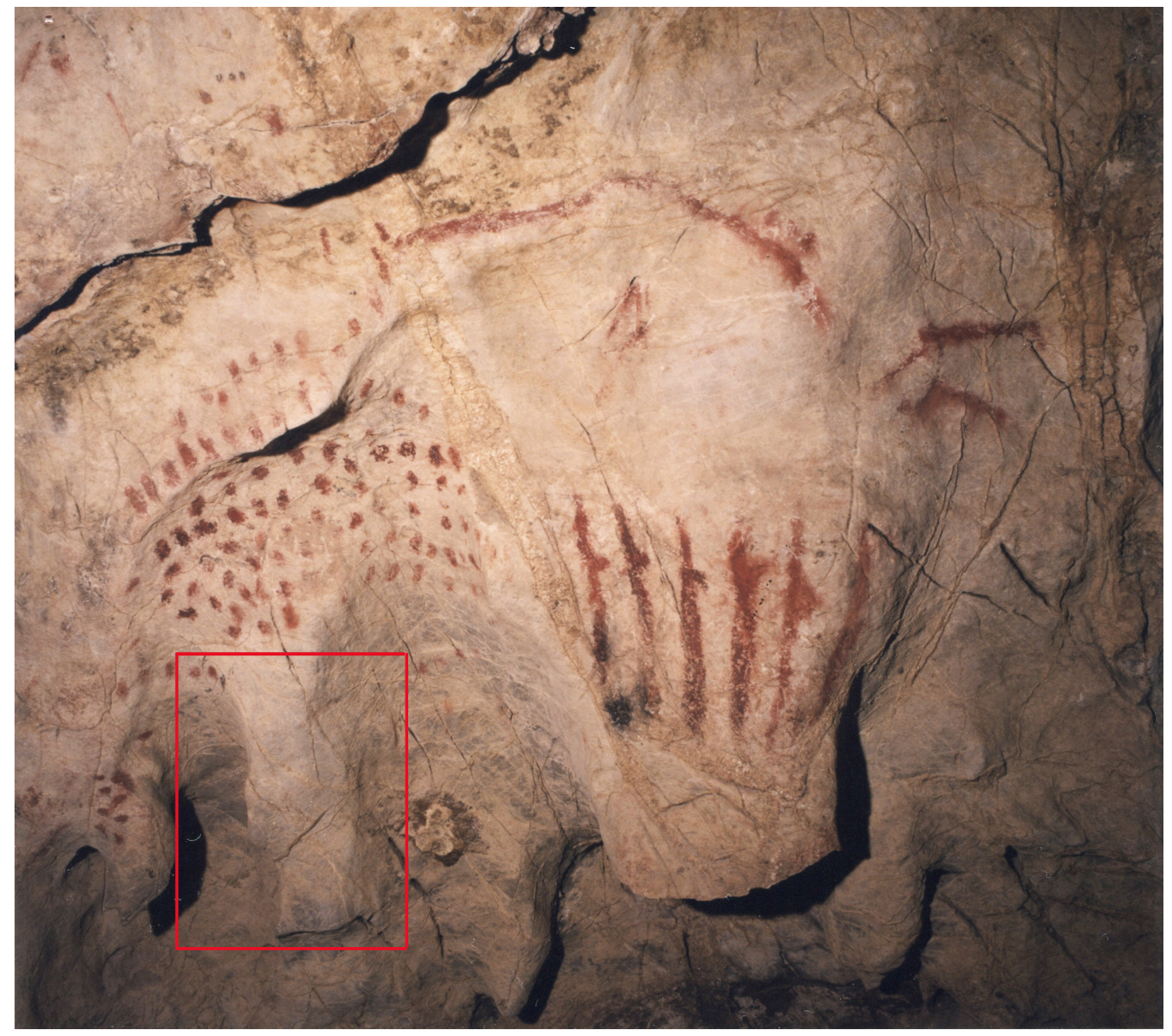

FIG. 7. El Pindal: Panel de los Claviformes. Recuadro rojo: localización de la cabeza de bisonte sobre la arista rocosa de la Fig. 6 (Foto: J. Fortea Pérez). 
fue mencionada por Balbín (Balbín et al., 1999) sin mencionar el ojo grabado que es lo que distingue un relieve aprovechado de un lusus naturae. La superficie de la roca, recubierta con viejas concreciones granulosas, contribuye a dar un aspecto natural a la cabeza, aunque sería ilusorio pretender identificar la especie. Por encima de la cabeza, y hacia atrás, hay dos trazos digitales rojos o, más bien, dos dedos frotados -MP$8-$, que no parecen en relación con ella. Aquella hipótesis que las interpreta como las astas de un reno (Balbín et al., 1999) es poco probable.

\section{Discusión}

Existen distintos tipos de utilizaciones de relieves en el arte paleolítico (Lejeune, 1985; Sauvet y Tosello, 1999; Martínez Bea, 2001-2002). Por un lado, los accidentes morfológicos, tales como cordones de calcita, estalactitas o grietas, que son aprovechados para representar algún detalle de la figura animal: un cuerno, una pata, un ojo, etc. Después, están las irregularidades más o menos marcadas, convexidades, concavidades o simples ángulos diedros, utilizadas

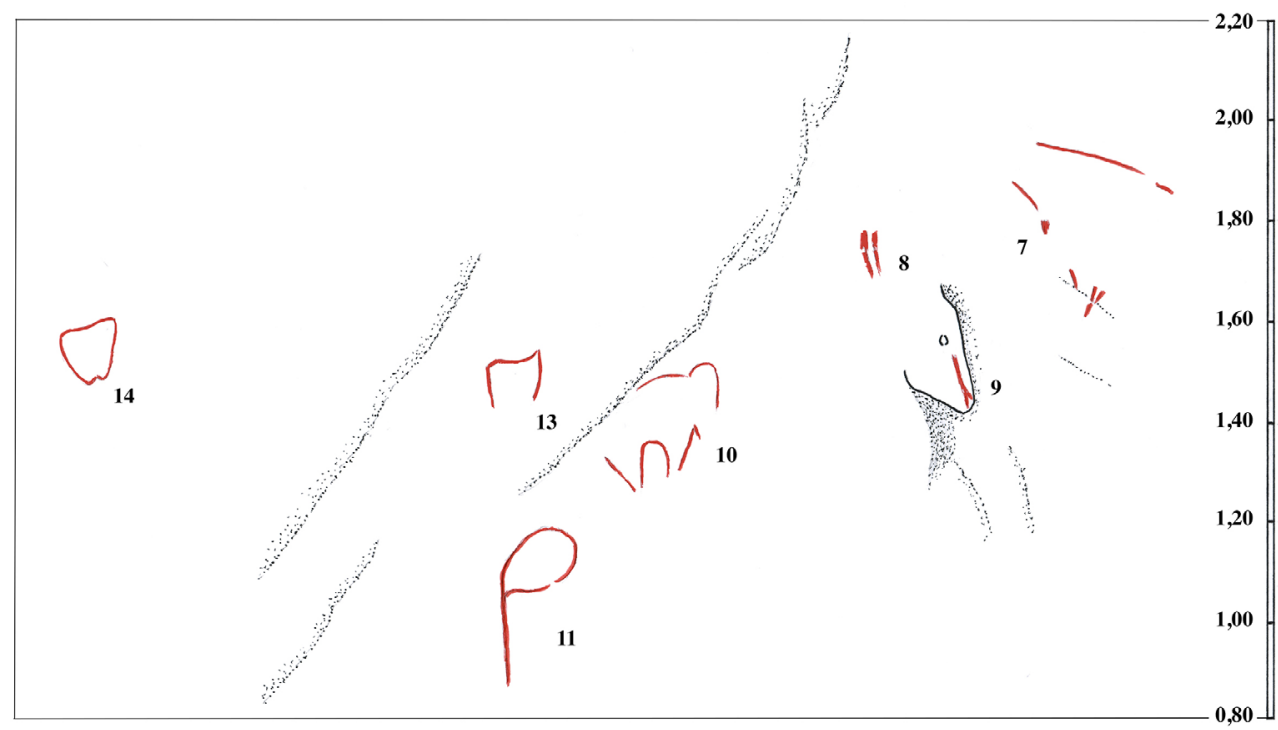

FIG. 8. El Pindal: dibujo del extremo derecho del panel principal (n. ${ }^{\text {s }}$ 7-14 del inventario de GonzálezPumariega, 2011).

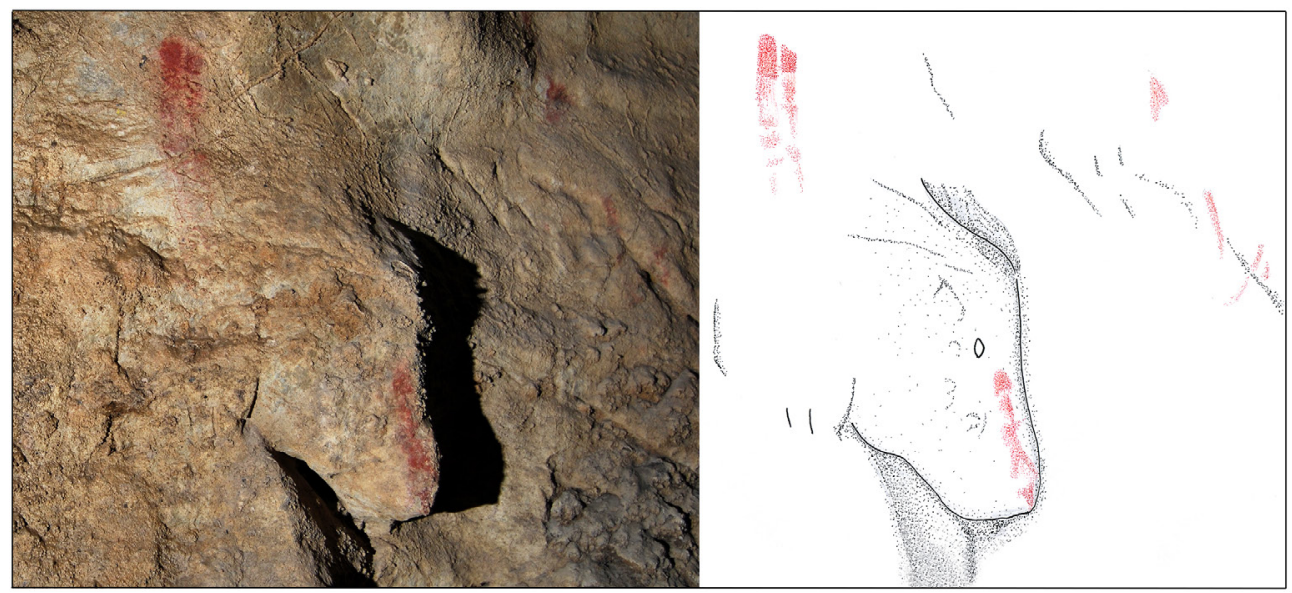

FIG. 9. El Pindal: resalte rocoso parcialmente separado de la pared, acondicionado como cabeza animal; cf. su localización en la Fig. 7. 

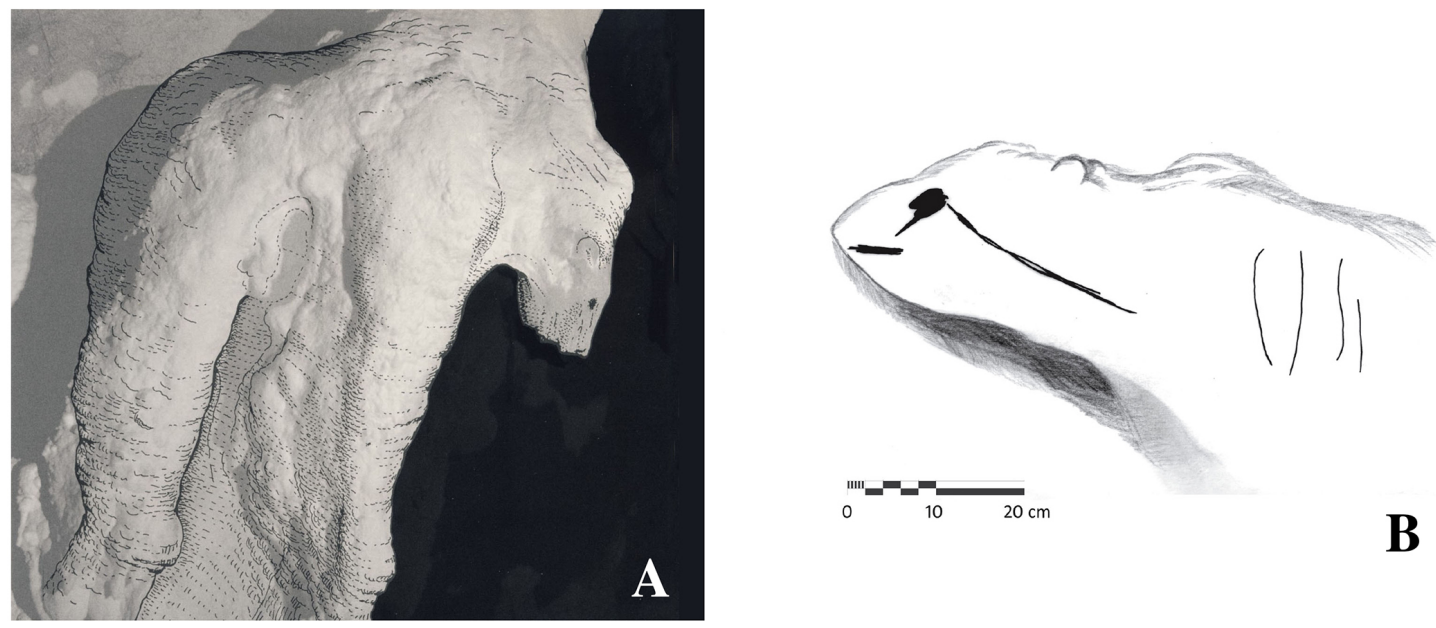

B
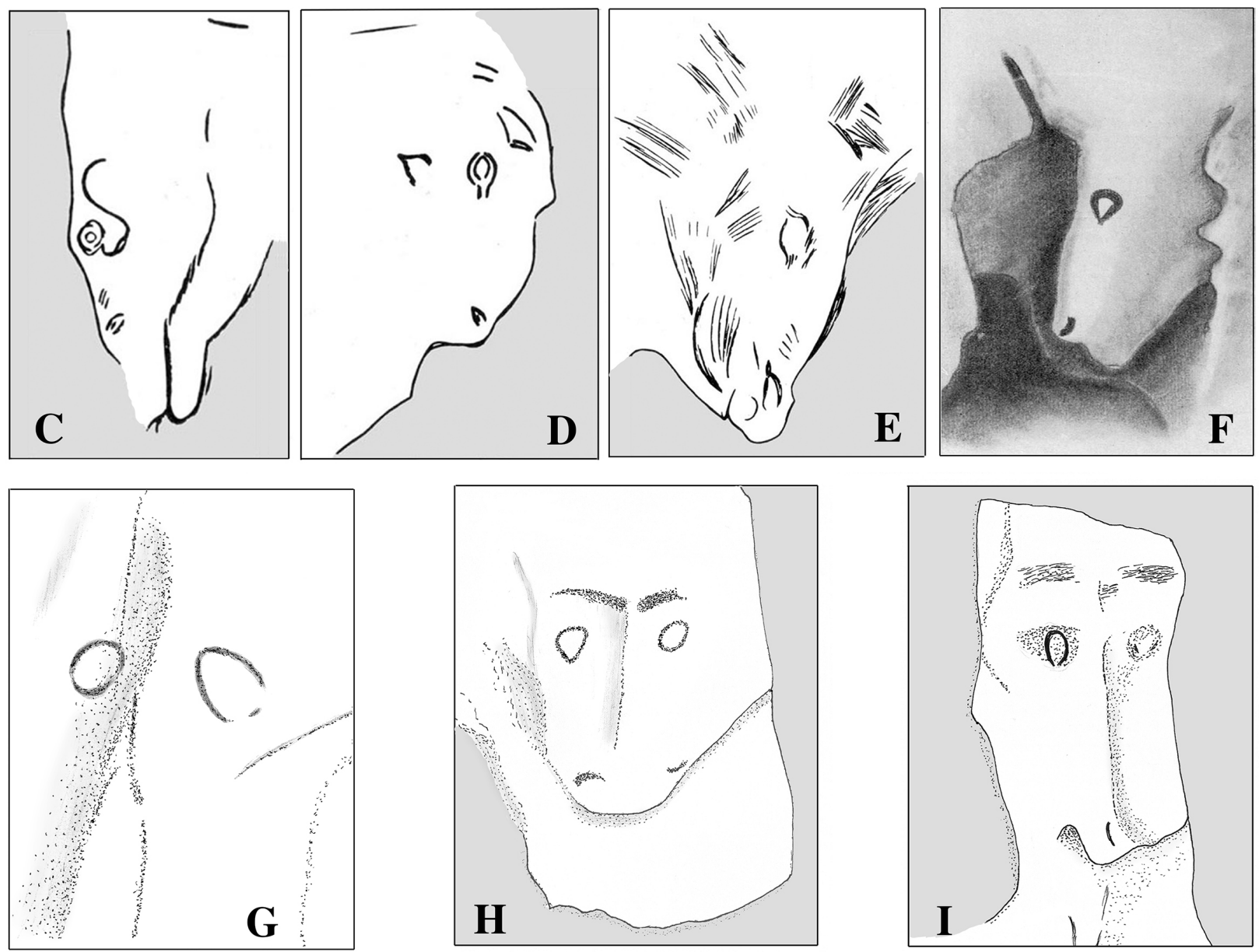

Fig. 10. Ejemplos de relieves acondicionados como figuras animales en las cuevas magdalenienses de Francia y España: A. Le Tuc d'Audoubert, Ariège (según Tosello); B. Bernifal, Dordoña (según Man-Estier); C-E. Les Trois-Frères, Ariège (según Breuil); F. El Castillo, Cantabria (según Ripoll); G. El Bosque, Asturias; H-I. Altamira, Cantabria (G-I a partir de fotografias). 
para colocar la giba de un bisonte o la crinera de un caballo. En ambos casos, los relieves o accidentes utilizados atañen sólo a una parte de la figura; no son suficientes para crear la ilusión visual de un animal, por lo que aquella debe ser necesariamente completada por un dibujo o un grabado, con el fin de concretar la intención figurativa. Un caso algo similar se encuentra en los pequeños agujeros hechos por el agua en la arcilla del suelo de la cueva de Niaux, que inspiraron un bisonte a un Magdaleniense: tres de ellos fueron interpretados como heridas y complementados por trazos grabados $\mathrm{y}$, un cuarto, figurado como ojo (Beltrán et al., 1973).

En muchas menos ocasiones, el relieve mismo se basta para evocar una forma animal. Así, las adiciones pintadas o grabadas no tienen por objeto hacer visible la figura, sino solamente mostrar que el autor la reconoció. Este parece ser el caso de los relieves de Bédeilhac y de El Pindal que acabamos de señalar. En ambos ejemplos, el autor economiza esfuerzos y se conforma con añadir ciertos trazos grabados o pintados, o bien un ojo colocado en posición correcta.

Este tipo de relieve transformado en cabeza animal, con mínimas adiciones, no es excepcional. Se conocen bastantes ejemplos, aunque generalmente juegan un papel secundario -Tuc d'Audoubert, Les Trois-Frères, Bernifal, Altamira, El Castillo, El Bosque, etc.-. Las 'máscaras' de la galería terminal de Altamira están entre las más célebres. Estos relieves, mínimamente acondicionados con la simple adición de un ojo, son raramente identificables (Fig. 10).

En la mayoría de los casos, la utilización de las irregularidades de la pared no es solo un artificio para crear la ilusión de la tercera dimensión y dar más realismo a la representación. El objetivo es, ciertamente, mucho más profundo. La pared no es considerada como un mero soporte sino que participa activamente de la puesta en escena de la composición gráfica. Parece que el establecimiento de una relación entre el animal y el mineral hubiera sido el motor de la creación artística, como si el fin fuera crear una verdadera simbiosis entre la obra y su soporte. Esto es particularmente evidente en el caso de Bédeilhac, donde la búsqueda sistemática de una relación entre los seres vivos figurados y la roca se muestra como motivación esencial tanto en el arte parietal como en el arte mueble. Esta relación parece vinculada a una concepción animista del universo, que explicaría la pregnancia -en el sentido de la Psicología de la Forma, cf. Arnheim, 2005otorgada a las formas de vida percibidas en la roca.

\section{Conclusiones}

Los dos ejemplos que acabamos de describir en esta nota sobre relieves naturales modificados para hacer surgir figuras animales de la pared muestran que incluso en cuevas conocidas desde hace más de un siglo, tales como Bédeilhac o El Pindal, quedan descubrimientos por hacer, particularmente cuando se trata de aspectos que pasan fácilmente desapercibidos para aquellos que no están preparados para reconocerlos, a diferencia de los magdalenienses.

La razón por la que este tipo de "utilización del relieve" es difícil de reconocer es, sin duda, de orden psicológico. En efecto, estos relieves siembran fácilmente la duda en el observador que teme verse afectado por el síndrome de la "piedra-figura". En Bédeilhac, particularmente, la frontera entre el lusus naturae y las creaciones magdalenienses es tenue. Podríamos pensar que el propio prehistórico experimentase esa dificultad y que, por ello, añadiera algunas señales - un ojo grabado, unos rastros de pigmento- con las que autentificar su descubrimiento, como si hacerlo realidad hubiera sido importante.

La especial atención que se concede a la representación del ojo para "hacer salir" a un animal de la roca es un aspecto recurrente. Expresa, sin duda, una motivación profundamente arraigada en las creencias que vinculan a los seres vivos con el mundo mineral, siendo el ojo el primer criterio identificador de los seres animados.

Desde el punto de vista antropológico, Bédeilhac y El Pindal se muestran como dos sitios parietales magdalenienses extraordinariamente próximos. Lo que ya es sabido por el estilo tan "pirenaico" de algunos caballos y bisontes grabados en la cueva asturiana, y sobre todo por la presencia de un grupo de 
claviformes típicos del Ariège, toma aún más fuerza si se añaden los relieves utilizados de una manera casi idéntica. Todo ello testimonia una comunidad de pensamiento, una misma percepción de la realidad y una misma concepción del universo. No queremos aventurarnos más allá en el campo especulativo de las creencias y de los ritos, pero podemos avanzar la idea de que a aquellos que frecuentaron el espacio subterráneo de Bédeilhac y de El Pindal les movían las mismas motivaciones. No se trata únicamente de meros intercambios, sino de una auténtica correspondencia cultural.

Finalmente, estos dos modestos relieves acondicionados tienen especial interés porque aportan luz sobre un aspecto de la mentalidad magdaleniense insuficientemente valorado, poniendo el acento sobre un comportamiento relevante frente al mundo subterráneo. Esta particular interacción que los magdalenienses mantuvieron con las paredes subterráneas es, sin duda, la clave de su atracción por el interior profundo de las cuevas. Si asumimos la hipótesis sobre las creencias de tipo animista, pareciera como si las paredes encerraran una vida latente que estuviera a la espera de que el hombre viniera a revelarla.

\section{Bibliografía}

Alcalde del Río, H.; Breuil, H. y Sierra, L. (1911): Les cavernes de la région cantabrique (Espagne). Monaco.

Álvarez Fernández, E. (2005): “Eloignés, mais pas isolés, la parure hors de la frontière française pendant le Magdalénien". En Dujardin, V. (ed.): Industrie osseuse et parures du Solutréen au Magdalénien en Europe. Mémoire spf, xxxix. Paris, pp. 25-38.

Arnheim, R. (2005): Arte y percepción visual: psicología del ojo creador. Madrid: Alianza Edit.

Balbín, R. de; Alcolea, J. J. y González Pereda, M. A. (1999): "Une vision nouvelle de la grotte de El Pindal, Pimiango, Ribadedeva, Asturies”, L'Anthropologie, 103 (1), pp. 51-92.
Beltrán, A.; Gailli, R. y Robert, R. (1973): La cueva de Niaux. Monografías arqueológicas, 16. Zaragoza.

González-Pumariega, M. (2005): "Una nueva figura de bisonte en la cueva de 'El Pindal' (Asturias): transformación de un relieve natural en una representación animal", Veleia, 22, pp. 21-26.

González-Pumariega, M. (2011): La cueva de El Pindal 1911-2011. Estudio de su arte rupestre cien años después de 'Les Cavernes de la région cantabrique'. Pola de Siero: Ménsula Ediciones.

Lejeune, M. (1985): "La paroi des grottes, premier 'mur' support artistique et document archéologique", Art et Fact, 4, pp. 15-24.

Martínez BeA, M. (2001-2002): "El aprovechamiento de accidentes naturales en el arte rupestre paleolítico: un nuevo caso en la cueva del Castillo (Puente Viesgo)", Salduie, II pp. 27-44.

Остовоn, Е. (1938): "Art et magie dans la grotte de Bédeilhac (Ariège)", Revue Anthropologique, 48, pp. 41-54.

Robert, R. (1946): "Fouilles à la grotte de Bédeilhac", Bulletin de la Société préhistorique française, 43 (912), pp. 322-326.

SAUvet, G. (2004): "L'art mobilier non classique de la grotte magdalénienne de Bédeilhac (Ariège)". En L'art du Paléolithique supérieur (Actes Colloque UISPP, Liège, 2001). ERAUL 107, pp. 167-176.

Sauvet, G. (2014): "Histoire de chasseurs. Chronique des temps paléolithiques". En Corchón, M. S. y Menéndez, M. (eds.): Cien años de arte rupestre paleolitico. Salamanca, pp. 15-30.

Sauvet, G.; Fortea, J.; Fritz, C. y Tosello, G. (2008): "Crónica de los intercambios entre los grupos humanos paleolíticos. La contribución del arte para el período 20000-12000 вр", Zephyrus, LXI, pp. 33-56.

Sauvet, G.; Fritz, C.; Fortea, J. y Tosello, G. (2014): "Fluctuations des échanges symboliques au Paléolithique supérieur en France et dans le Nord de l'Espagne". En Jaubert, J.; Fourment, N. y Depaepe, P. (eds.): Transitions, ruptures et continuité en Préhistoire (Actes Congrès Préhistorique de France, Bordeaux-Les Eyzies, 2010), pp. 403-416.

Sauvet, G. y Tosello, G. (1999): "Le mythe paléolithique de la caverne". En Sacco, G. y Sauvet, G. (eds.): Le Propre de l'Homme. Lausanne: Delachaux et Niestlé, pp. 55-90. 
